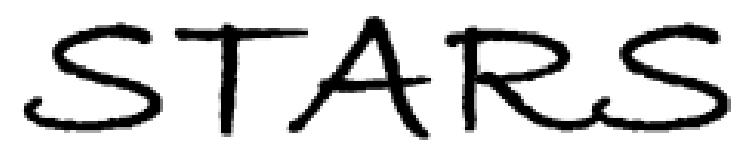

University of Central Florida

STARS

$1-1-1994$

\title{
Base And Collector Leakage Currents Of Algaas/Gaas Heterojunction Bipolar-Transistors
}

\author{
J. J. Liou \\ University of Central Florida \\ C. I. Huang \\ B. Bayraktaroglu \\ D. C. Williamson \\ K. E. Parab \\ University of Central Florida
}

Find similar works at: https://stars.library.ucf.edu/facultybib1990

University of Central Florida Libraries http://library.ucf.edu

This Article is brought to you for free and open access by the Faculty Bibliography at STARS. It has been accepted for inclusion in Faculty Bibliography 1990s by an authorized administrator of STARS. For more information, please contact STARS@ucf.edu.

\section{Recommended Citation}

Liou, J. J.; Huang, C. I.; Bayraktaroglu, B.; Williamson, D. C.; and Parab, K. E., "Base And Collector Leakage Currents Of Algaas/Gaas Heterojunction Bipolar-Transistors" (1994). Faculty Bibliography 1990s. 2960.

https://stars.library.ucf.edu/facultybib1990/2960

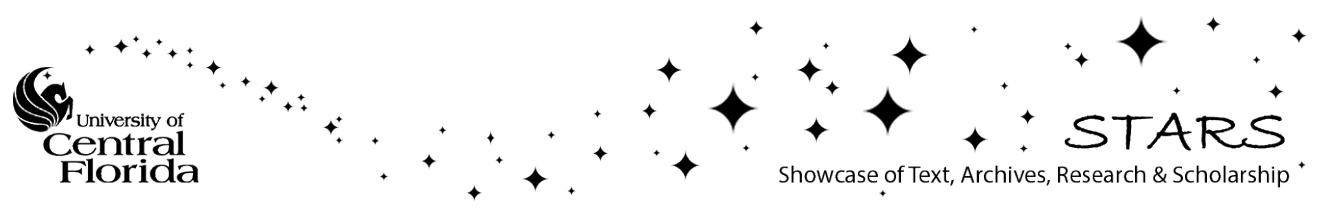




\section{Base and collector leakage currents of AlGaAs/GaAs heterojunction bipolar transistors}

Cite as: Journal of Applied Physics 76, 3187 (1994); https://doi.org/10.1063/1.357502

Submitted: 16 December 1993 . Accepted: 22 April 1994 . Published Online: 17 August 1998

J. J. Liou, C. I. Huang, B. Bayraktaroglu, D. C. Williamson, and K. B. Parab

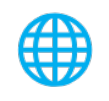

\section{ARTICLES YOU MAY BE INTERESTED IN}

Heterojunction bipolar transistor using pseudomorphic GalnAs for the base Applied Physics Letters 49, 179 (1986); https://doi.org/10.1063/1.97217

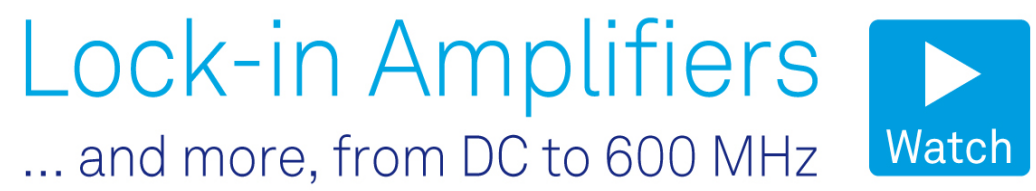




\title{
Base and collector leakage currents of AIGaAs/GaAs heterojunction bipolar transistors
}

\author{
J. J. Liou \\ Electrical and Computer Engineering Department, University of Central Florida, Orlando, Florida 32816 \\ C. I. Huang and B. Bayraktaroglu \\ Solid State Electronic Directorate, Wright Laboratory, Wright-Patterson AFB, Ohio 45433-7323 \\ D. C. Williamson \\ Electromagnetic and Reliability Directorate, Rome Laboratory, Griffiss AFB, New York 13422-4505 \\ K. B. Parab \\ Electrical and Computer Engineering Department, University of Central Florida, Orlando, Florida 32816
}

(Received 16 December 1993; accepted for publication 22 April 1994)

\begin{abstract}
Base and collector leakage currents are extremely important to AlGaAs/GaAs heterojunction bipolar transistor (HBT) dc characteristics, and a simple model to describe such currents is presented. This study suggests that these currents are originated from the electron and hole leakage through the dielectric-layer (e.g., polyimide, nitride, etc.) interface at the emitter-base and base-collector peripheries, as well as through the $n^{+}$-subcollector/semi-insulating substrate interface. Five HBTs having similar intrinsic make-ups (i.e., doping concentration and layer thickness) but different extrinsic make-ups (i.e., finger pattern, perimeter, dielectric layer, etc.) are investigated, and with the aid of the model, the possible mechanisms contributing to their leakage behavior identified.
\end{abstract}

\section{INTRODUCTION}

Base and collector leakage currents are the dominant components of the base and collector currents, respectively, of AlGaAs/GaAs heterojunction bipolar transistors (HBTs) operated at relatively small bias voltages. Such currents have been widely observed in experimental measurements, and a good understanding of their origin and physical mechanisms is useful to HBT design. In addition, since the leakage currents are directly related to properties of the HBT peripheries, which can be degradable under stressful conditions (e.g., high-level injection, high temperature, etc.), their trend and magnitude have important implications on the HBT reliability, such as the current gain and $1 / f$ noise long-term instability.

Despite the extensive studies of the other current components, such as the space-charge region recombination current, ${ }^{1}$ surface recombination current, ${ }^{2,3}$ and base bulk recombination current, ${ }^{4}$ the properties of leakage currents have largely been overlooked in the literature.

This paper develops the first analytical model to characterize the base and collector leakage currents in $N / p / n$ $\mathrm{AlGaAs} / \mathrm{GaAs}$ HBTs. For a given device geometry and make-up, the model can satisfactorily explain the biasdependent behavior of the two leakage currents. Five HBTs having similar intrinsic make-ups (i.e., doping concentration and layer thickness) but different extrinsic make-ups (i.e., finger pattern, perimeter, dielectric layer, etc.) are investigated, and with the aid of the model, the possible mechanisms contributing to their leakage behavior identified.

\section{BASE AND COLLECTOR LEAKAGE CURRENTS}

The structure of a typical mesa-etched, single-emitter finger HBT is shown in Fig. 1. The base and collector leak- age currents can take place at the emitter-base perimeter (circle 1 in Fig. 1), base-collector perimeter (circle 2), collector-subcollector perimeter (circle 3), and subcollectorsubstrate interface (circle 4). The magnitude of such currents depends strongly on the etching process, the quality of the emitter-base and base-collector peripheries which is covered by the dielectric (e.g., polyimide, nitride, etc.) layer, and the quality of $n^{+}-\mathrm{GaAs} / \mathrm{semi}$-insulating GaAs interface. For discussion, we assume the HBT surface is protected with a polyimide layer. An inferior quality emitter and base peripheries can thus increase the possibility for the free carriers to either surmount or tunnel through the GaAs-polyimide and AlGaAs-polyimide interface barriers and subsequently increase the leakage currents. ${ }^{5}$ As will be shown later, the peripheral quality is insensitive to the type of dielectric layer, but rather, varies strongly from process to process.

\section{A. Leakage currents at emitter-base periphery}

The base and collector leakage currents originated at the emitter perimeter $\left(I_{B L, E}\right.$ and $\left.I_{C L, E}\right)$ are affected by the applied base-emitter voltage $V_{B E}$. As $V_{B E}$ is increased, the potential barriers at the GaAs-polyimide and AlGaAspolyimide interfaces are lowered, thus increasing the numbers of electrons in the $n$-type AlGaAs and holes in the $p$-type GaAs to surmount the barriers and reach the base and emitter regions, respectively. Once there, these excess minority carriers are no different from those injected across the emitter-base heterojunction and thereby contribute additional base and collector currents.

The leakage currents can be modeled using the physical degradation mechanism described in the Arrhenius relationship, ${ }^{6}$ which expresses the physicochemical reaction rate as 


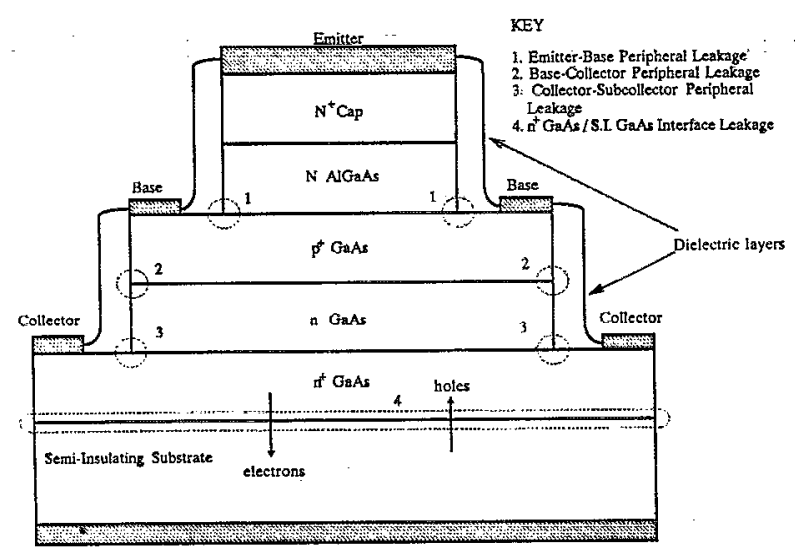

FIG. 1. Schematic illustration of the cross section of a typical HBT structure, with leakage peripheries circled.

$$
k_{1}=k_{10} \exp \left(-E_{a} / k T\right),
$$

where $k_{1}$ is the reaction rate, $k_{10}$ is a constant peculiar to the reaction type, $E_{a}$ is the activation energy of the degradation process, $k$ is the Boltzmann constant, and $T$ is the absolutc temperature. This relation suggests that the degradation rate decreases sharply as $E_{a}$ is increased. The total activation rate $k_{t}$ is obtained by integrating the right-hand side of (1) from $E_{a}=0$ to the highest activation energy $E_{a}^{\prime}$ :

$$
k_{t}=k^{\prime}\left[1-\exp \left(-E_{a}^{\prime} / k T\right)\right],
$$

where $k^{\prime}$ is a constant related to $k_{10}$.

Based on the above concept, $I_{B L, E}$ and $I_{C L, E}$ can be expressed analogous to (2) as

$$
\begin{aligned}
& I_{B L, E}=N J_{B L, E} P_{E}\left[1-\exp \left(-V_{B E} F_{L} / V_{T}\right)\right], \\
& I_{C L, E}=N J_{C L, E} P_{E}\left[1-\exp \left(-V_{B E} F_{L} / V_{T}\right)\right],
\end{aligned}
$$

where $N$ is the number of emitter fingers, $J_{B L, E}$ is the leakage hole current density $(\mathrm{A} / \mathrm{cm})$ from the base to emitter, $J_{C L, E}$ is the leakage electron current density from the emitter to base, $P_{E}$ is the emitter perimeter length $\left[P_{E}=2\left(W_{E}+L_{E}\right)\right.$, where $W_{E}$ and $L_{E}$ are the emitter finger width and length, respectively], $F_{L}$ is an empirical parameter, and $V_{T}=k T / q$ is the thermal voltage. The values of $J_{B L, E}, J_{C L, E}$, and $F_{L}$ depend on the process. Equations (3) and (4) suggest that the leakage currents taking place at the emitter periphery increase very rapidly and become near constant as $V_{B E}$ is increased. This trend will later be verified with experimental results.

\section{B. Leakage currents at base-collector periphery}

Since the base-collector junction is reverse biased under forward-active operation, a large electric field exists in the nitride near the base and collector periphery. This high electric field gives rise to a large probability for the electrons in the $p$-type GaAs and holes in the n-type GaAs to tunnel through the GaAs-polyimide potential barrier and reach the collector and base regions, respectively. Similar to the electrons and holes generated from avalanche multiplication in the base-collector depletion region, ${ }^{7}$ the electrons entering the collector increase the collector current, whereas the holes entering the base are forced to flow out of the base terminal, which constitutes a negative current component and reduces the total base current.

Following the same approach as that in the previous section, the base and collector leakage currents occurring at the base perimeter $\left(I_{B L, B}\right.$ and $\left.I_{C L, B}^{*}\right)$ can be modeled as

$$
\begin{aligned}
& I_{B L, B}=J_{B L, B} P_{B}\left[1-\exp \left(-V_{C B} F_{L} / V_{T}\right)\right], \\
& I_{C L, B}=J_{C L, B} P_{B}\left[1-\exp \left(-V_{C B} F_{T} / V_{T}\right)\right],
\end{aligned}
$$

where $J_{B L, B}$ is the leakage hole current density from the collector to base, $J_{C L, B}$ is the leakage electron current density from the base to collector, $P_{B}$ is the base perimeter length, and $V_{C B}$ is the applied collector-base voltage. For a multifinger HBT, $P_{B}$ is a function of the emitter finger geometry as well as the finger spacing

$$
P_{B}=2\left\{\left[N W_{E}+(N+1) S_{1}\right]+\left(L_{E}+2 S_{2}\right)\right\},
$$

where $S_{1}$ and $S_{2}$ are the horizontal and vertical spacings of the emitter finger.

\section{Leakage currents at the collector-subcollector periphery}

When subjected to an applied voltage, the $n^{+} / n$ periphery, similar to the $n / p$ base-collector periphery, can inject free carriers through the interface of dielectric layer covering the periphery and thus give rise to both base and collector leakage currents. These leakage currents have the same bias dependence as $I_{B L, B}$ and $I_{C L, B}$. However, since the voltage drop of the applied base-collector voltage occurs primarily at the $p / n$ base-collector junction, the voltage drop at the $n^{+} / n$ high-low junction is negligibly small. ${ }^{8}$ As a result, the leakage current at the $n^{+} / n$ periphery, which is proportional to $\left[\exp \left(V^{\prime} / V_{T}\right)-1\right]\left(V^{\prime}\right.$ is the voltage drop across the highlow junction), can be neglected.

\section{Leakage currents through the subcollector- substrate interface}

Another leakage current is the leakage of free carriers through the $n^{+}$-GaAs/SI-GaAs (SI denotes semi-insulating) interface. Such an interface is "leaky" due to the very high defect density at the SI GaAs surface, and kinetic factors such as the chemical barrier and diffusion rate often prevent the system from reaching the equilibrium state. ${ }^{9}$ Since the SI substrate has a lower electron density and higher hole density than the $n^{+}$subcollector, electrons and holes can leak, or diffuse, through the nonequilibrium interface and enter the SI substrate and the subcollector, respectively. Because of the high defect density in the SI substrate, electrons entering the SI substrate will recombine with holes via the defect centers. To maintain charge neutrality in the $n^{+}$subcollector, electrons are then supplied into the subcollector from the collector contact, which constitutes a current flow $I_{C L, S I}$ opposite to the normal collector current flow. Thus, such a leakage mechanism can be represented by including a negative collector current component $\left(-I_{C L, S I}\right)$ in the collector current model. 
TABLE I. HBT emitter finger pattern and geometry. All HBTs have very similar intrinsic device make-up such as the doping concentrations and layer thicknesses.

\begin{tabular}{lclccccc}
\hline \hline Device & No. of finger & Finger shape & $\begin{array}{c}\text { Finger area } \\
\left(\mu \mathrm{m}^{2}\right)\end{array}$ & $\begin{array}{c}\text { Spacing } \\
(\mu \mathrm{m})\end{array}$ & $\begin{array}{c}N \times P_{E} \\
(\mu \mathrm{m})\end{array}$ & $\begin{array}{c}P_{B} \\
(\mu \mathrm{m})\end{array}$ & Dielectric layer \\
\hline HBT-1 & $5 \times 5$ & circular & 7 & 10 & 235 & 300 & polyimide \\
HBT-2 & 6 & rectangular & 25 & 10 & 150 & 230 & nitride \\
HBT-3 & 6 & rectangular & 25 & 10 & 150 & 230 & nitride \\
HBT-4 & 3 & rectangular & 40 & 10 & 132 & 172 & nitride \\
HBT-5 & 4 & rectangular & 50 & 10 & 180 & 200 & nitride \\
\hline \hline
\end{tabular}

The effects of the $n^{+}$-GaAs/SI-GaAs leakage on the base current can be more conveniently treated by focusing on the hole transport. Once entering the $n^{+}$region, the holes, which are minority carriers, will diffuse across the $n^{+}$and $n$ regions. Depending on the recombination process in these regions, a percentage of the holes will reach the basecollector depletion region and be swept into the base region by the large electric field at the base-collector junction. Since the number of holes allowed to be injected into the emitter is fixed by the emitter-base voltage, these extra holes are forced to flow out of the base terminal, which constitutes a current flow opposite to the normal base current flow. Thus, a negative base current component $\left(-I_{B L, S I}\right)$ also needs to be included in the base current model to account for the $n^{*}$-GaAs/SI-GaAs interface leakage. Obviously, to maintain quasineutrality in the SI substrate, holes need to be supplied from a current path through the grounded substrate.

Unlike the leakage process at the emitter-base and basecollector peripheries which require the free carriers to surmount or tunnel through the potential barrier associated with the dielectric layer, the leakage at the subcollector/substrate interface is caused by the diffusion of electrons and holes through the leaky interface. As a result, $I_{B L, S I}$ and $I_{C L, S I}$ are independent of the applied voltage.

\section{E. Normal base and collector currents}

The normal base current $I_{B N}$ consists of (1) the recombination current $I_{S C R}$ in the emitter-base space-charge region; (2) the surface recombination current $I_{R S}$ at the emitter sidewalls and extrinsic base surface; (3) the recombination current $I_{R B}$ in the quasineutral base region; and (4) the injection current $I_{R E}$ from the base into emitter. Thus

$$
\begin{aligned}
I_{B N}= & I_{S C R}+I_{R S}+I_{R B}+I_{R E} \\
= & I_{1} \exp \left(V_{B E} / 2 V_{T}\right)+I_{2} \exp \left(V_{B E} / V_{T}\right) \\
& +I_{n}\left(X_{2}\right)(1-\alpha)+I_{p}\left(X_{1}\right) \exp \left(-V_{B} / V_{T}\right),
\end{aligned}
$$

where $I_{1}$ and $I_{2}$ are the pre-exponential currents for $I_{S C R}$ and $I_{R S}$, respectively, $I_{n}\left(X_{2}\right)$ is the electron current at the edge of the quasineutral base, $I_{p}\left(X_{1}\right)$ is the hole current at the edge of the quasineutral emitter, $\alpha$ is the base transport factor, and $V_{B}$ is the valence-band barrier potential across the emitterbase junction. Note that $I_{1}$ is determined by the ShockleyRead-Hall recombination process, $I_{2}$ is a function of the surface states and location of Fermi-level pinning, $\alpha$ is influenced by the carrier mobility and lifetime in the base, and
$I_{n}\left(X_{2}\right)$ and $I_{p}\left(X_{1}\right)$ depend on the emitter and base doping concentrations, layer thicknesses, as well as the heterojunction properties. It is important to point out that, unlike all other base current components which are directly proportional to the emitter area, $I_{2}$ is proportional to the surface area and thus does not scale with the emitter area. ${ }^{10}$

The normal collector current $I_{C N}$ is given by

$$
I_{C N}=I_{n}\left(X_{2}\right) \alpha .
$$

Note that $I_{C N}$ is affected by the carrier transport across the heterojunction, which for an abrupt heterojunction is governed by the thermionic-tunneling-diffusion mechanism. The drift-diffusion theory becomes applicable if the conductionband spike is effcctivcly removed by grading the $\mathrm{Al}$ composition near the heterointerface.

From the preceding discussions, the total base and collector currents $\left(I_{B}\right.$ and $\left.I_{C}\right)$ model including the leakage currents at the emitter-base periphery, base-collector periphery, and $n^{+}-\mathrm{GaAs} / \mathrm{SI}-\mathrm{GaAs}$ interface are

$$
\begin{aligned}
& I_{B}=I_{B N}+I_{B L, E}-I_{B L, B}-I_{B L, S I} \\
& I_{C}=I_{C N}+I_{C L, E}+I_{C L, B}-I_{C L, S I} .
\end{aligned}
$$

\section{RESULTS AND DISCUSSIONS}

To support the foregoing phenomenological reasoning and modeling, we consider $5 \mathrm{AlGaAs} / \mathrm{GaAs}$ HBTs (HBT-1HBT-5) having very similar intrinsic make-ups (i.e., doping concentration and layer thickness) but different extrinsic make-ups (i.e., finger pattern, perimeter, dielectric layer, etc.) and different manufactures. Table I gives the detailed finger pattern including the finger number, shape, spacing, as well as the emitter and base perimeter lengths and type of dielectric layer covering the HBT surface.

Figure 2(a) shows the Gummel plots of HBT-1 calculated from the present model and obtained from measurements at $V_{C B}=0$. For this device, empirical parameters $J_{B L, E}=8.3 \times 10^{-6} \mathrm{~A} / \mathrm{cm}, \quad J_{C L, E}=1.1 \times 10^{-5} \mathrm{~A} / \mathrm{cm}$, $F_{L}=0.005$, and $I_{B L, S I}=I_{C L, S I}=0$ are used in calculations to give the observed leakage current versus $V_{B E}$ characteristics. The different $J_{B L, E}$ and $J_{C L, E}$ may arise from the asymmetrical properties of AlGaAs polyimide and GaAs-polyimide interfaces at the emitter perimeter. The fact that using $I_{B L, S I}=I_{C L, S I}=0$ fits the data well, indicates that such a device has a good (nonleaky) $n^{+}$-GaAs/SI-GaAs interface. 


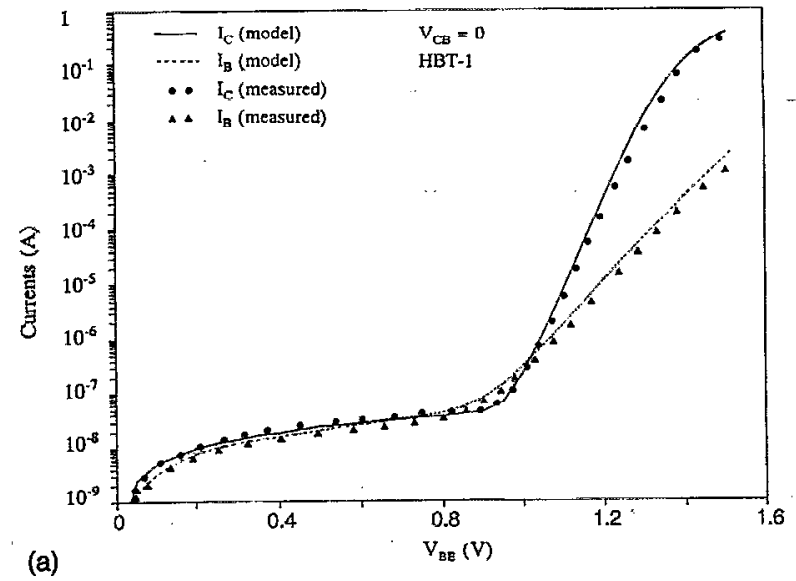

(a)

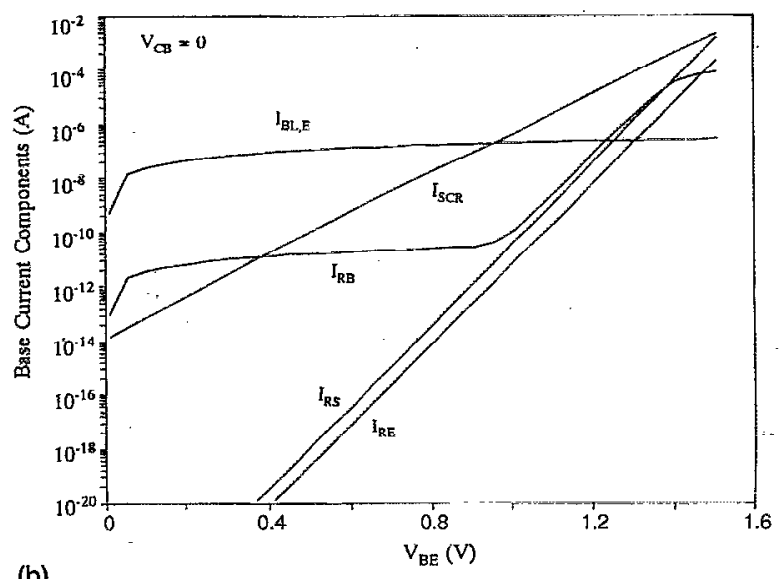

(b)

FIG. 2. (a) Base and collector currents calculated from the present model and obtained from measurements for HBT-1 at $V_{C B}=0$; (b) the corresponding base current components.

Note that $I_{B L, B}=I_{C L, B}=0$ for this case because $V_{C B}=0$ [Eqs. (5) and (6)], which leads to $I_{B} \approx I_{C}$ at small $V_{B E}$.

The relative importance of the base current components at $V_{C B}=0$ is illustrated in Fig. 2(b). Clearly, the base leakage current $I_{B L, E}$ is the dominant component for $V_{B E}<0.8 \mathrm{~V}$. For small $V_{B E}, I_{R B}$ has the same shape as $I_{B L, E}$. This is because $I_{R B}$ is proportional to $I_{C}$ [Eq. (8)], which in turn is equal to $I_{C L, E}\left(\approx I_{B L, E}\right)$ under such bias conditions. As $V_{B E}$ increases, $I_{B L, E}$ becomes negligibly small and $I_{R B}$ returns to its $V_{T}$ like slope. The surface recombination current $I_{R S}$, like $I_{R E}$, also possesses a $V_{T}$-like slope ${ }^{10}$ and becomes more important as $V_{B E}$ is increased. On the other hand, $I_{S C R}$ has a $2 V_{T}$-like slope due to the dominance of the Shockley-ReadHall recombination process in the space-charge region.

Figure 3(a) shows the Gummel plots of HBT-1 at $V_{C E}=2.5 \mathrm{~V}$. Note that $V_{C B}\left(=V_{C E}-V_{B E}\right)$ varies with $V_{B E}$ in this figure. In addition to the leakage current densities at the emitter perimeter mentioned earlier, $J_{B L, B}=7 \times 10^{-6}$ $\mathrm{A} / \mathrm{cm}$ and $J_{C L, B}=4 \times 10^{-5} \mathrm{~A} / \mathrm{cm}$ are also used here to describe the base perimeter leakage. It should be pointed out that assuming $J_{B L, B}=J_{C L, B}$ in calculations will result in $I_{B}=I_{C}$ at $V_{B E}=0$ for all $V_{C E}$ [see Eqs. (10) and (11)], a trend not supported by measurement data [see Fig. 3(a)]. Under the bias condition considered in Fig. 3(a), the leakage current at the base perimeter is larger than that at the emitter

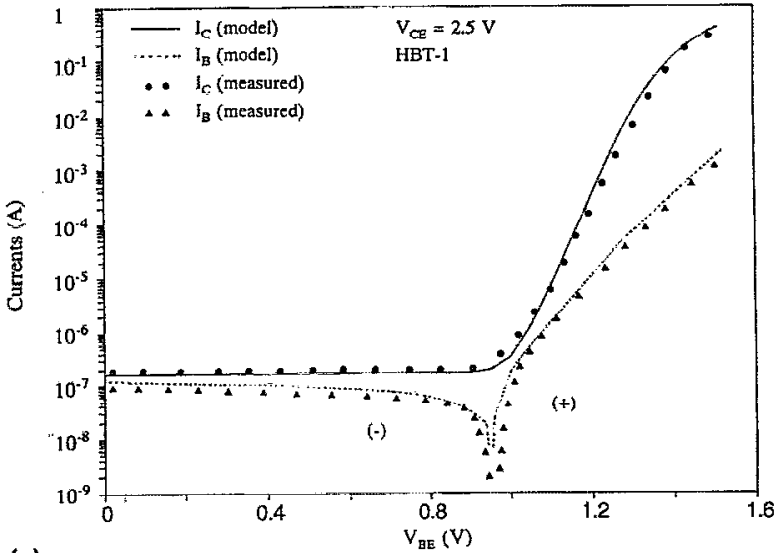

(a)

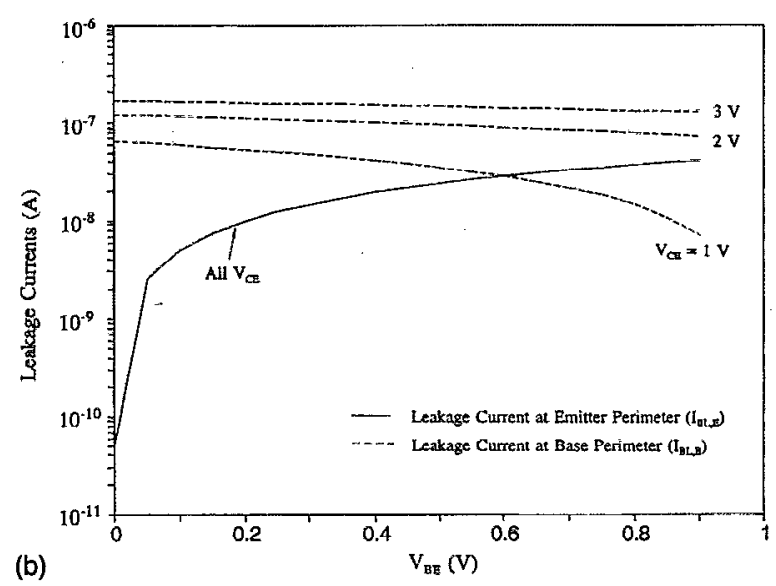

FIG. 3. (a) Base and collector currents calculated from the present model and obtained from measurements for HBT-1 at $V_{C E}=2.5 \mathrm{~V}$; (b) leakage currents vs $V_{B E}$ calculated for three different $V_{C E}$.

perimeter $\left[I_{B L, B}>I_{B L, E}\right.$ and $I_{C L, B}>I_{C L, E}$ in Eqs. (10) and (11)]. This is evidenced in the results shown in Fig. 3(b). For $V_{C E}>0$ and small $V_{B E}$, the larger leakage at the base perimeter than emitter perimeter, together with the negligibly small $I_{B N}$ and $I_{C N}$, yields the observed negative base current (current flow is in the direction opposite to that of the normal flow) and near constant collector current.

Figures 4(a) and 4(b) show the $I_{C}$ and $I_{B}$ characteristics calculated from the model and obtained from measurements of HBT-2 at $V_{C B}=0$ and $V_{C E}=2.5 \mathrm{~V}$, respectively. Constant values of $I_{B L, S I}=I_{C L, S I}=5 \times 10^{-8} \mathrm{~A}$ are used in calculations, suggesting that HBT-2 has a leaky $n^{+}$-GaAs/SI-GaAs interface. This leakage which leads to a trend differs considerably from that seen in HBT-1. Both $I_{B}$ and $I_{C}$ have a dip at $V_{B E} \approx 1 \mathrm{~V}$ and $V_{C B}=0\left(I_{B}\right.$ and $I_{C}$ are negative for $V_{B E}<1$ V). At $V_{C E}=2.5 \mathrm{~V}$, however, only $I_{B}$ has a dip, and $I_{C}$ is positive for all $V_{B E}$. Equally important to note is that while HBT-1 and HBT-2 have different types of dielectric layer, they nonetheless have very similar leak current densities at the emitter and base peripheries (see Table II). This suggests that the leakage mechanism at the peripheries is insensitive to the type of passivation layer, but rather is strongly affected by the property of the interface which varies from process to process. Conversely, it is likely that HBTs having the same type of dielectric layer can have considerable different leak- 


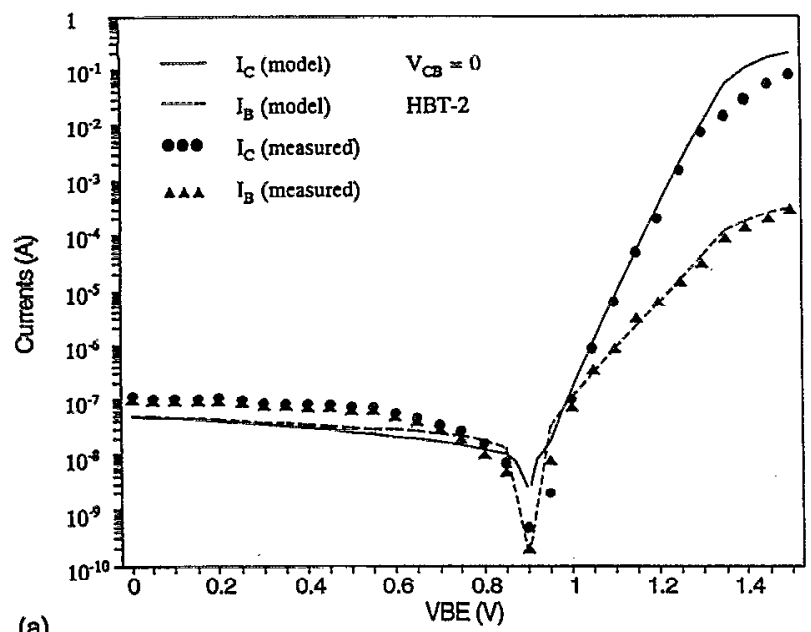

(a)

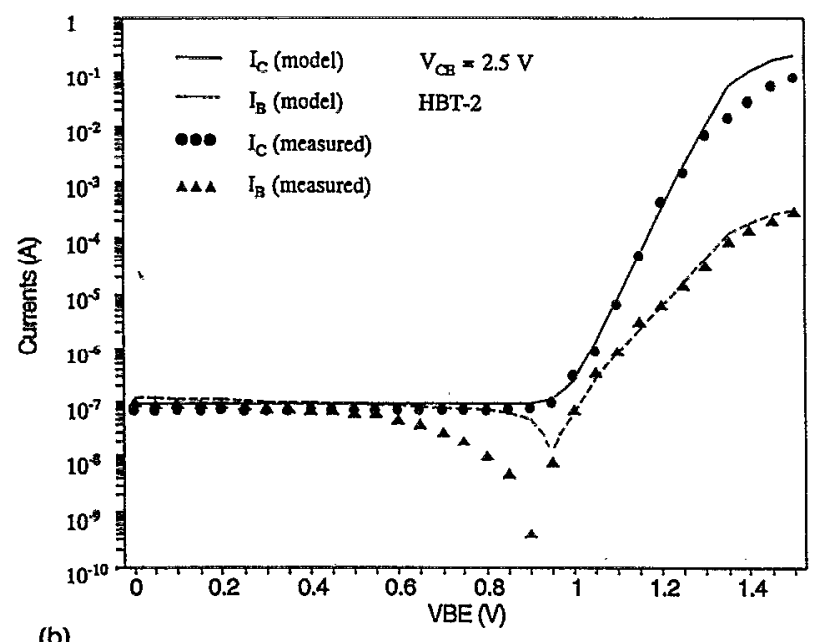

(b)

FIG. 4. Base and collector currents calculated from the present model and obtained from measurements for HBT-2 at (a) $V_{C B}=0$ and (b) $V_{C E}=2.5 \mathrm{~V}$.

age current characteristics. This point is evidenced by the leakage current densities of HBT-2-HBT-5 (all have nitride layers) given in Table II.

We now turn our attention to another HBT (HBT-3). This device has the same finger pattern and geometry as HBT-2, but was fabricated from a different lot. Compared to HBT-2, HBT-3 has a very similar $I_{C}$ behavior but very different $I_{B}$

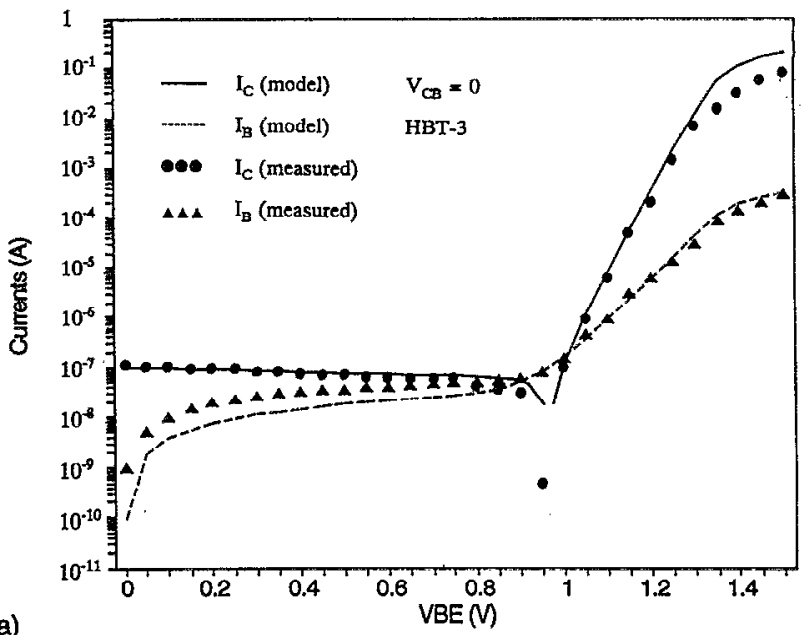

(a)

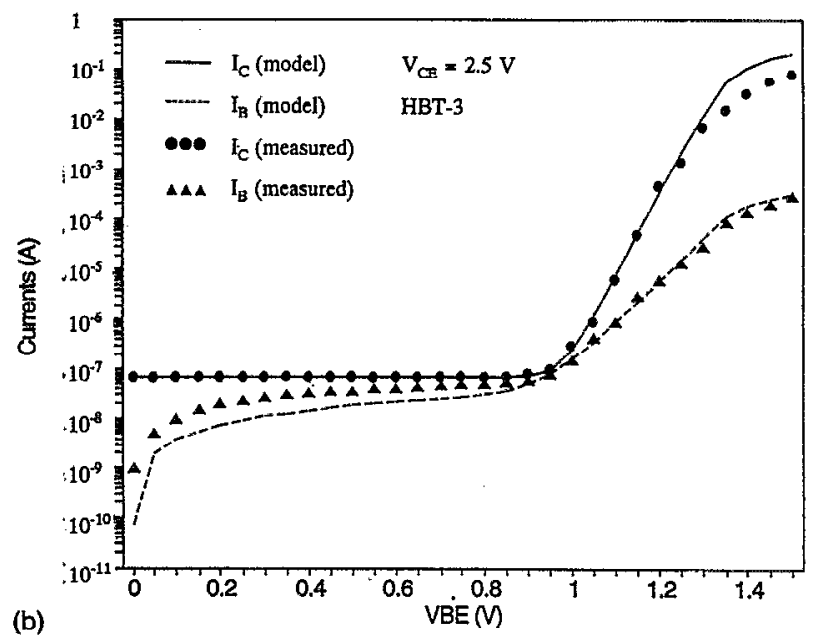

FIG. 5. Base and collector currents calculated from the present model and obtained from measurements for HBT-3 at (a) $V_{C B}=0$ and (b) $V_{C E}=2.5 \mathrm{~V}$.

characteristics, as shown in Figs. 5(a) and 5(b). The present model can still be used to describe such $I_{B}$ and $I_{C}$ characteristics, provided $I_{B L, S I}$ and $I_{B L, B}$ are neglected in model calculations. The large $I_{C L, S I}$ and zero, or very small, $I_{B L, S I}$ required in calculations may arise from the fact that this HBT has a leaky subcollector/substrate interface as well as a high electron-hole recombination process in collector and subcol-

TABLE I. Leakage current components used in calculations.

\begin{tabular}{lllllll}
\hline \hline Device & $J_{B L, E}(\mathrm{~A} / \mathrm{cm})$ & $J_{C L, E}(\mathrm{~A} / \mathrm{cm})$ & $J_{B L, B}(\mathrm{~A} / \mathrm{cm})$ & $J_{C L, B}(\mathrm{~A} / \mathrm{cm})$ & $I_{B L, S I}(\mathrm{~A})$ & $I_{C L, S I}(\mathrm{~A})$ \\
\hline HBT-1 $^{\mathrm{a}}$ & $8.3 \times 10^{-6}$ & $1.1 \times 10^{-5}$ & $7.0 \times 10^{-6}$ & $1.4 \times 10^{-5}$ & 0.0 & 0.0 \\
HBT-2 $^{\mathrm{b}}$ & $1.3 \times 10^{5}$ & $1.7 \times 10^{-5}$ & $9.0 \times 10^{-6}$ & $1.8 \times 10^{-5}$ & $5.0 \times 10^{-8}$ & $5.0 \times 10^{-8}$ \\
HBT-3 $^{\mathrm{c}}$ & $1.3 \times 10^{-5}$ & $1.7 \times 10^{-5}$ & 0.0 & 0.0 & 0.0 & $5.0 \times 10^{-8}$ \\
HBT-4 $^{\mathrm{d}}$ & $5.0 \times 10^{-4}$ & $4.0 \times 10^{-4}$ & $1.0 \times 10^{-4}$ & $5.0 \times 10^{-4}$ & $5.0 \times 10^{-8}$ & $5.0 \times 10^{-8}$ \\
HBT-5 $^{\mathrm{c}}$ & $3.5 \times 10^{-4}$ & $7.0 \times 10^{-4}$ & $4.2 \times 10^{-4}$ & $1.1 \times 10^{-3}$ & 0.0 & 0.0 \\
\hline
\end{tabular}

${ }^{2}$ HBT-1: average emitter-base periphery, average base-collector periphery, good subcollector/substrate interface. bHBT-2: average emitter-base periphery, average base-collector periphery, leaky subcollector/substrate interface.

'HBT-3: average emitter-base periphery, good base-collector periphery, leaky subcollector/substrate interface and substantial electron-hole recombination in the collector and subcollector.

${ }^{d}$ HBT-4: poor emitter-base periphery, poor base-collector periphery, leaky subcollector/substrate interface.

'HBT-5: poor emitter-base periphery, poor base-collector periphery, good subcollector/substrate interface. 


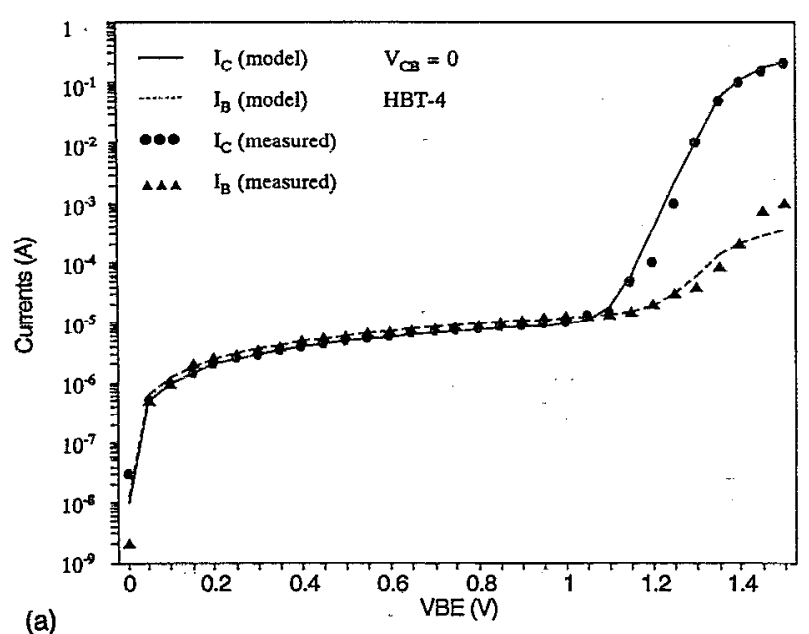

(a)

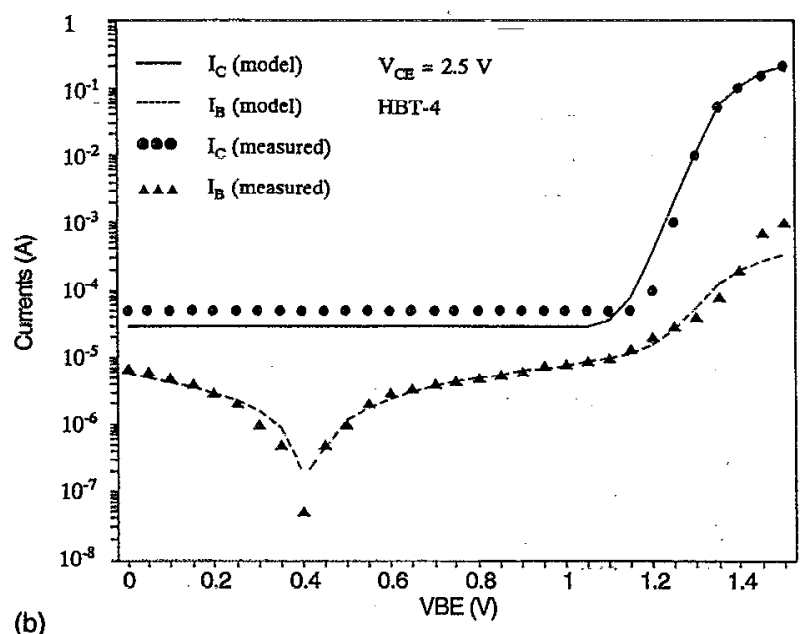

FIG. 6. Base and collector currents calculated from the present model and obtained from measurements for HBT-4 at (a) $V_{C B}=0$ and (b) $V_{C E}=2.5 \mathrm{~V}$.

lector. As a result, the vast majority of holes leak from the substrate into the subcollector are recombined with electrons in the $n^{+}$and $n$ regions before they reach the base region. On the other hand, the negligible $I_{B L, B}$ may stem from a good quality base-collector perimeter of the HBT, thus minimizing the leakage at the periphery. The different trends obtained from the identical HBT-2 and HBT- 3 illustrate that the HBT lcakage currents are highly process dependent.

Two more HBTs (HBT-4 and HBT-5) having similar finger pattern and geometry as HBT-3 but fabricated from different manufactures are also investigated, and their leakage behavior successfully described by the model, as shown in Figs. 6(a) and 6(b) and Figs. 7(a) and 7(b), respectively. Fitting the model and measurements, we found that both devices have relatively large leakage current densities at the base and base peripheries (see Table II). Their different leakage behavior shown in Figs. 6 and 7 arises from the fact that the subcollector/substrate interface leakage is minimal in HBT-5 and is large in HBT-4.

From the foregoing analysis, it can be seen that the HBT leakage currents are affected by the six current components in a complex kind of manner, particularly for HBTs exhibiting negative base and/or collector currents (i.e., HBT-2,

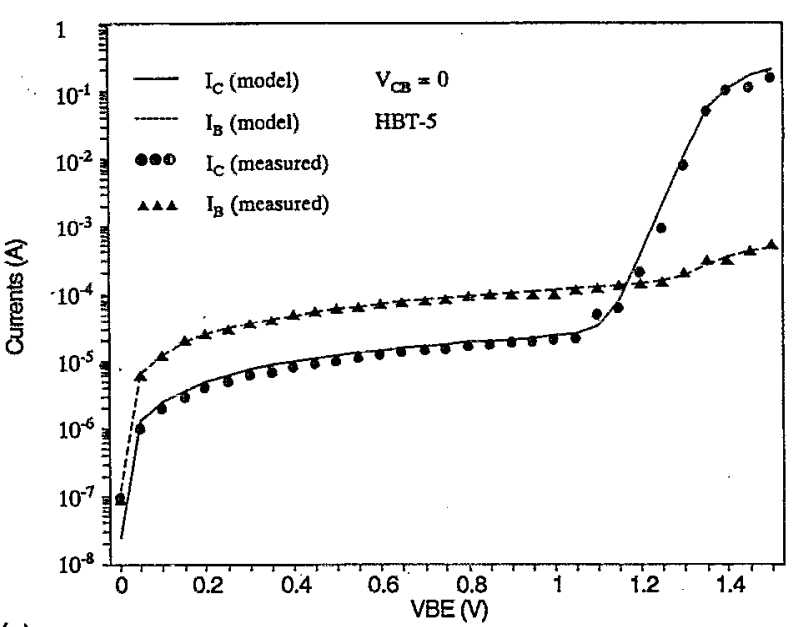

(a)

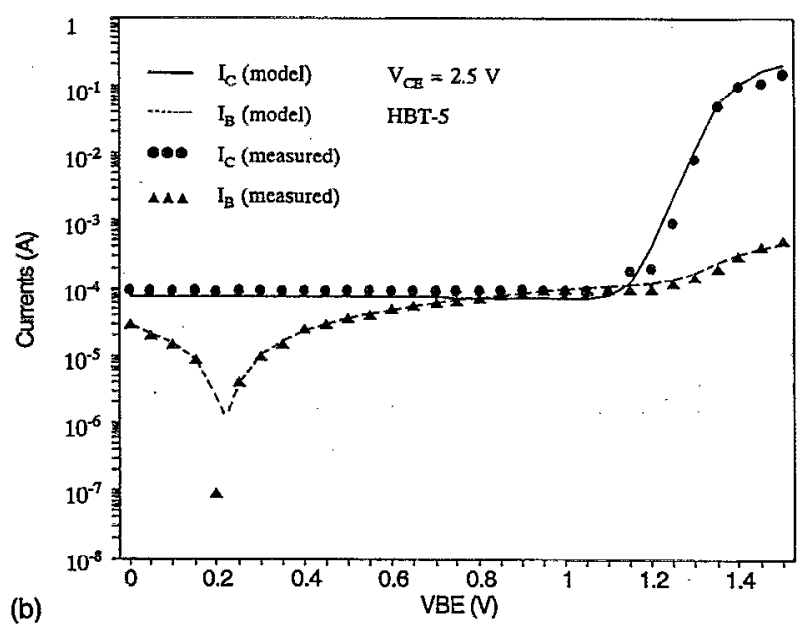

FIG. 7. Base and collector currents calculated from the present model and obtained from measurements for HBT-5 at (a) $V_{C B}=0$ and (b) $V_{C E}=2.5 \mathrm{~V}$.

HBT-3, and HBT-4). Here, we summarize the modeling procedure to make the determination of leakage current components more efficient. Since there is only one negative current component (the leakage current $I_{C L, S I}$ due to the leaky $n^{+}$ $\mathrm{GaAs} / \mathrm{SI} \mathrm{GaAs}$ interface) in the total collector current [Eq. (11)], the negative collector leakage current can be attributed to $I_{C L, S I}$. The positive portion of the collector leakage current is then fitted by the two leakage current components $\left(I_{C L, E}\right.$ and $\left.I_{C L, B}\right)$ occurred at the emitter and base perimeters. The base leakage current, on the other hand, is more complicated than its collector counterpart because it involves two negative $\left(I_{B L, B}\right.$ and $\left.I_{B L, S I}\right)$ and one positive components $\left(I_{B L, E}\right)$ [Eq. (10)]. When a negative base current is observed in measurement at $V_{C B}=0, I_{B L, S I}$ is used in the model to give a best fitting to the experimental data. The other negative component $I_{B L, B}$, which is a function of $V_{C B}$, is then used to fit the negative base current at $V_{C B}>0$ (i.e., $V_{C E}=2.5 \mathrm{~V}$ ). The positive portion of the base leakage current is fitted using $I_{B L, E}$. If negative base and collector leakage currents are absent at $V_{C B}=0$, then $I_{B L, S I}=I_{C L, S I}=0$, as for the case of HBT-1 and HBT-5.

Table II summarizes the leakage current components used in model calculations to fit the experimental data. Based 
on these values, comments on the property of the HBT's emitter-base periphery, base-collector periphery, and subcollector-substrate interface are also given (see Table II).

\section{CONCLUSION}

The base and collector leakage currents in the AlGaAs/ GaAs HBTs are studied, and an analytical model which can fully describe such currents is developed. It is found that the leakage currents take place at the emitter-base periphery, base-collector periphery, and subcollector-substrate interface. Five HBTs having different extrinsic make-ups (i.e., finger pattern, perimeter, and dielectric layer) and having been fabricated by different manufactures are investigated, and different leakage current characteristics are found. With the aid of the model developed, the physical mechanisms responsible for the observed leakage behavior are identified.

This study should have important implications in correlating the observed current-voltage characteristics and the peripheral and interface properties of $\mathrm{AlGa} \mathrm{As} / \mathrm{GaAs}$ HBTs. Such a correlation is useful to the prediction and analysis of the HBT reliability, e.g., the current gain and $1 / f$ noise longterm instability.

${ }^{1}$ C. D. Parikh and F. A. Lindholm, IEEE Trans. Electron Devices 39, 2197 (1992).

${ }^{2}$ C. J. Sandroff, R. N. Nottengurg, J. C. Bischoff, and R. Bhat, Appl. Phys. Lett. 51, 33 (1987).

${ }^{3}$ S. Tiwari, D. J. Frank, and S. L. Wright, J. Appl. Phys. 64, 5009 (1988). ${ }^{4}$ W. Liu, D. Costa, and J. S. Harris, Solid-State Electron. 34, 119 (1991).

${ }^{5}$ D. E. Aspnes, Surf. Sci. 132, 406 (1983).

${ }^{6}$ M. S. Ash and H. C. Gorton, IEEE Trans. Reliability 38, 485 (1989).

${ }^{7}$ J. J. Liou and J. S. Yuan, IEEE Trans. Electron Devices 37, 2274 (1990).

${ }^{8}$ R. M. Warner, Jr. and B. L. Grung, Transistors: Fundamentals for the Integrated-Circuit Engineer (Wiley, New York, 1983), Chap. 6.

${ }^{9} \mathrm{C}$. W. Wilmsen, in The Physics of Submicron Semiconductor Devices, edited by H. L. Grubin et al. (Plenum, New York, 1988).

${ }^{10}$ W. Liu and J. S. Harris; Jr., IEEE Trans. Electron Devices 39, 2726 (1992). 